Published in final edited form as:

J Child Psychol Psychiatry. 2008 November ; 49(11): 1193-1200. doi:10.1111/j.

1469-7610.2008.01944.x.

\title{
Evidence for three subtypes of repetitive behavior in autism that differ in familiality and association with other symptoms
}

\author{
Kristen S.L. Lam ${ }^{1}$, James W. Bodfish ${ }^{1,2}$, and Joseph Piven ${ }^{1,2}$ \\ ${ }^{1}$ Neurodevelopmental Disorders Research Center, University of North Carolina - Chapel Hill, NC, \\ USA \\ ${ }^{2}$ Department of Psychiatry, University of North Carolina - Chapel Hill, NC, USA
}

\section{Abstract}

\begin{abstract}
Background-Restricted repetitive behaviors (RRBs) are a core feature of autism and consist of a variety of behaviors, ranging from motor stereotypies to complex circumscribed interests. The objective of the current study was to examine the structure of RRBs in autism using relevant items from the Autism Diagnostic Interview-Revised in a sample of 316 individuals with autistic disorder.
\end{abstract}

\begin{abstract}
Methods/Results-Using exploratory factor analysis, three distinct factors were identified: Repetitive Motor Behaviors (RMB), Insistence on Sameness (IS), and Circumscribed Interests (CI). RMB were found to be associated with a variety of subject characteristics such as IQ, age, social/communication impairments, and the presence of regression. IS was associated with social and communication impairments whereas $\mathrm{CI}$ appeared to be independent of subject characteristics, suggesting CI may be particularly useful in subsetting samples. Based on sib-pair correlations, IS and $\mathrm{CI}$ (but not RMB) appear to be familial. Analysis of the data at the case level suggests that the presence of multiple forms of RRB in an individual is associated with more impairment in the social and communication domains, suggesting a more severe presentation of autistic disorder.
\end{abstract}

Conclusions-There appears to be considerable structure within repetitive behavior in autism. The finding that these behaviors are differentially related to subject characteristics and familiality adds to their validity.

\section{Keywords}

Autism; repetitive behavior; factor analysis; Autism Diagnostic Interview-Revised

\begin{abstract}
Autism is a pervasive developmental disorder characterized by social impairments, deficits in verbal and nonverbal communication, and repetitive interests and behaviors (American Psychiatric Association, 1994; World Health Organization, 1992). Each of these core features must be present in order to diagnose autism; however, the severity and clinical presentation of autism is extremely heterogeneous. It is now widely accepted that autism is a neurobiological disorder with a strong genetic basis (e.g., Folstein \& Piven, 1991; Lotspeich \& Ciaranello, 1993; Rutter, 2005). Although genetic investigation has revealed potential autism susceptibility genes, there has been very little replication across studies (VeenstraVanderweele, Christian, \& Cook, 2004). It is likely that the clinical complexity of autism
\end{abstract}

(C) 2008 The Authors Journal compilation @ 2008 Association for Child and Adolescent Mental Health.

Correspondence to: Kristen S.L. Lam, CB\# 3367, University of North Carolina - Chapel Hill, Chapel Hill, NC 27599-3367, USA; Tel: (919) 966-2382; Fax: (919) 843-3825; kristen_lam@med.unc.edu.

Conflict of interest statement: No conflicts declared. can best distinguish autism from other disorders 
has constrained our ability to consistently identify specific genetic factors for the disorder; it is also possible that genetic heterogeneity in these samples has impeded efforts to replicate previous findings.

One way to facilitate genetic research is to identify more homogenous subgroups based on phenotypic characteristics. For example, several groups have provided evidence for linkage in certain chromosomal areas using age of first words or phrases to stratify samples (Buxbaum et al., 2001; Bradford et al., 2001; Wassink et al., 2004; Shao et al., 2002). While these findings are promising, recent work has also shown that age of language acquisition is confounded with other subject characteristics, such as IQ and overall severity of autism (Hus, Pickles, Cook, Risi, \& Lord, 2007). Ideally, one would hope to subset groups by a relatively independent set of behaviors that do not co-segregate with other phenotypic characteristics.

Recent work has suggested that restricted repetitive behaviors (RRBs) may be used to reveal homogenous subgroups within autism. Once considered to be a single, unitary domain of behavior, there is growing evidence that there is considerable structure within RRBs. In the Diagnostic and Statistical Manual of Mental Disorders - 4th Edition (DSM-IV; American Psychiatric Association, 1994), criteria for RRB can be met by a person exhibiting at least one of the following: '(a) encompassing preoccupation with one or more stereotyped and restricted patterns of interest that is abnormal either in intensity or focus; (b) apparently inflexible adherence to specific, nonfunctional routines or rituals; (c) stereotyped and repetitive motor mannerisms (e.g., hand or finger flapping or twisting or complex wholebody movements); or (d) persistent preoccupation with parts of objects.' What becomes clear upon examination of these criteria is that they are very broad, ranging from repetitive movements of the body to more cognitively-mediated symptoms such as intense interests or hobbies. Although no single type of RRB may be specific to autism, previous studies have found that it is a pattern of multiple types of RRB that can best distinguish autism from other disorders (Bodfish, Symons, Parker, \& Lewis, 2000; Bartak \& Rutter, 1976).

In a recent study of 307 individuals with autism spectrum disorders (ASDs), Lam and Aman (2007) examined the empirical structure of repetitive behaviors as measured by the Repetitive Behavior Scale-Revised (RBS-R; Bodfish et al., 2000), an informant-based questionnaire designed for use in autism. Results from a factor analysis suggested that there were 5 distinct types of repetitive behavior within ASDs. To date, several studies (Cuccaro et al., 2003; Shao et al., 2003; Bishop, Richler, \& Lord, 2006, Szatmari et al., 2006) have also examined the structure of RRBs as measured by the Autism Diagnostic InterviewRevised (ADI-R, Rutter, Le Couteur, \& Lord, 2003). These studies have identified two factors: a 'lower-order' category called 'Repetitive Sensory and Motor Behaviors' (RSMB), and a 'higher-order' category labeled 'Insistence on Sameness' (IS). Evidence for familial aggregation of the IS (but not the RSMB) factor was shown (Szatmari et al., 2006; Shao et al., 2003). Recently,Hus et al. (2007) examined the IS factor and found that relative to the RSMB factor, the IS factor was relatively independent of other subject characteristics (such as age and IQ) and, unlike other behavioral groupings such as RSMB or age of language acquisition, it was found to be somewhat independent. This suggests that higher-order RRBs may be a useful way to subset groups in genetic analyses.

While previous studies of the ADI-R have yielded similar 2-factor solutions for the RRB domain, closer examination of the items included (and excluded) from the factors suggest that there may be more structure present. In particular, in the solutions provided byCuccaro et al. (2003) andSzatmari et al. (2006), the ADI-R items 'Unusual Preoccupations,' 'Unusual Attachments,' and 'Circumscribed Interests' do not load on either factor and are therefore excluded. These types of RRB may be of particular interest in autism, because unlike motor 
stereotypies and compulsions (which are found in other disorders such as obsessive compulsive disorder, Tourette's syndrome, and mental retardation), these behaviors may be particularly characteristic of autistic disorder. In addition, the 2-factor solutions have accounted for a small amount of variance in each study (between 32 and 36\%), suggesting the presence of other factors. Lastly, Szatmari and colleagues used principal components analyses (PCA) to describe the structure of RRBs. PCA is best viewed as a data reduction tool; components are linear combinations of observed variables. A better way to reveal latent constructs within this domain is to use exploratory factor analysis (EFA), which is designed to examine theoretical structure of behavioral domains (Fabrigar, Wegener, MacCallum, \& Strahan, 1999).

The purpose of the current study was to examine the structure of the repetitive behavior symptom domain in autism as measured by the ADI-R using EFA. In addition, the validity of RRB subtypes was assessed by examining their relation with other subject characteristics, such as age, IQ, and severity of autism. The familiality of the different domains of RRB also was assessed using examination of sib-pair data. Lastly, cases were sorted based on factor scores to see if grouping by RRBs reveals homogenous subgroups in autism that may aid genetic and neurobiological investigation.

\section{Method}

\section{Sample}

Our sample included 316 individuals with autistic disorder who were consenting participants in genetic and/or imaging studies. Subjects were recruited from: North Carolina (primarily from 9 specialty clinics for pervasive developmental disorders; Treatment and Education of Autistic and Related Communication Handicapped Children [TEACCH] Centers); Iowa (University of Iowa Child Psychiatry Clinics); and Maryland (John Hopkins University) (Barrett et al., 1999; Hazlett, Poe, Gerig, Smith, \& Piven, 2006; Hazlett et al., 2005; Piven et al., 1997). All subjects included in the study met criteria for autistic disorder on the ADI-R. In addition, participants met criteria of autistic disorder using both the Autism Diagnostic Observational Schedule (ADOS; Lord, Rutter, DiLavore, \& Risi, 2002) and DSM- IV (American Psychiatric Association, 1994) criteria. Individuals with identifiable chromosomal or neurological conditions (with the exception of seizures) were excluded. Subjects were from both singleton and multiplex families; if siblings were included in the data base, one sibling was randomly selected for inclusion in the factor analysis. The resulting sample was $82.5 \%$ male, ranged in age from 20 months to 29 years (mean $=9.02$ years, $\mathrm{SD}=6.15$ ), and was predominately Caucasian $(85 \%)$. Data on IQ was available for 160 individuals and ranged from 20 to 133 (mean $=69.5, \mathrm{SD}=23.3$ ); a small proportion of the sample $(n=10)$ functioned in the severe to profound range of mental retardation (IQ < 40).

\section{Instruments}

The Autism Diagnostic Interview-Revised or ADI-R (Lord, Rutter, \& Le Couteur, 1994) is a standardized parent interview designed to assess the presence and severity of symptoms based on the DSM-IV criteria for autism in three diagnostic categories: social relatedness, communication, and restricted range of interests and behaviors. Two scores are given for most items: a 'current' score, which assesses behavior in the past 3 months, and an 'ever' score, which assesses behavior in early childhood or at its greatest severity. The ADI-R has demonstrated validity across a wide range of ages and severity levels. Administration was conducted by examiners who have been trained to research reliability (90\%) on the ADI-R via the University of Michigan model. 
Ten ADI-R items which assess restricted repetitive behaviors were included in the analysis. These items were identical to those used by Cuccaro et al. (2003) and Szatmari et al. (2006), with the exception of Item 71 'Unusual Sensory Interests.' We excluded this item from our analyses as sensory features of autism are not formally included as part of the restricted repetitive domain (DSM-IV; American Psychiatric Association, 1994). Sensory symptoms are considered associated features of ASD (DSM-IV; American Psychiatric Association, 1994) and appear to be strongly related to mental age (Baranek, David, Poe, Stone, \& Watson, 2006). The relation between sensory symptoms and RRB is unclear. We used the 'current' behavior ratings so that age effects could be examined and to avoid any potential retrospective recall bias that could result from using the 'ever' behavior ratings. The full range of scores (0 to 3 ) were used for the analyses.

The Autism Diagnostic Observation Schedules or ADOS (Lord et al., 2002) is a structured observational assessment administered directly to participants. It is employed to confirm behaviors reported on the ADI-R and consists of four different modules, each adapted for a different level of language ability. Algorithm scores for each of the modules have high levels of specificity and sensitivity to discriminate children with autism from those with other developmental disorders.

Measurement of cognitive performance-For 59 subjects, the Mullen (1995) Scales of Early Learning were administered directly by our research staff. Cognitive data was acquired for an additional 104 subjects by reviewing medical and educational records, and included the following instruments: Wechsler Adult Intelligence Scale-Revised (WAIS-R; Wechsler, 1981); Wechsler Intelligence Scale for Children-3rd Edition (WISC-III; Wechsler, 1991); Leiter International Performance Scales (Arthur, 1952).

\section{Statistical analyses}

In order to replicate the methods of previous studies, an initial principal components analysis (PCA) with varimax rotation was run on the $10 \mathrm{RRB}$ items from the ADI-R using SPSS 14.0. Using the results from the PCA as a guide, a subsequent exploratory factor analysis (EFA) was run using generalized least squares discrepancy function in CEFA (comprehensive exploratory factor analysis) with target rotation. Target rotation is a confirmatory procedure where the user constructs a target matrix which specifies the general pattern of loadings expected. Using EFA with target rotation is similar to confirmatory factor analysis (CFA) in that one has an idea of the number of factors and where items will load, though in CFA certain loadings are forced to be zero. In EFA target rotation loadings are made as close to zero as possible, but are not forced, so one can tell if the data doesn't support the theory or target (Browne, 2001).

The effect of subject characteristics on factor scores were examined using Pearson correlations (for continuous variables) and analysis of variance (ANOVAs) for categorical variables. To assess familiality, the factor scores of sib-pairs were examined using intraclass correlations (ICCs). Lastly, four subgroups of cases were created based on level and amount of RRB (see below). Differences between groups on social and communication impairments were analyzed using ANOVAs.

\section{Results}

\section{Factor structure}

Descriptive statistics for the 10 ADI-R items used in these analyses can be found in Table 1. An initial PCA with varimax rotation was performed, and solutions with 2, 3, and 4 components were examined. The number of components to retain was guided by: (a) the 
scree plot method (Cattell, 1966), (b) eigenvalues above 1.0, and (c) interpretability. Using these criteria, a three-component solution was selected, which has a simple structure in that the high component loadings were high and the other loadings were low (see Table 2). Each component contained three items, and were as follows: (I) Repetitive Motor Behaviors (RMB), which included Item 72 'Repetitive Use of Objects,' Item 81 'Hand and Finger Mannerisms,' and Item 84 'Other Complex Mannerisms/Stereotyped Body Movements'; (II) Insistence on Sameness (IS), which included Item 73 'Difficulties with Minor Changes in Personal Routine and Environment,' Item 74 'Resistance to Trivial Changes in the Environment,' and Item 75 'Compulsions and Rituals'; and (III) Circumscribed Interests (CI), which included Item 70 'Circumscribed Interests,' 1 Item 71 'Unusual Preoccupations,' and Item 76 'Unusual Attachment to Objects.' Overall, this three-component solution accounted for $52 \%$ of the variance.

The EFA showed the same three-factor solution and item pattern loadings as the PCA (see Table 2). The root mean square error of approximation (RMSEA; Browne \& Cudeck, 1992) point estimate for this three-factor solution was .000, and the upper bound of the $90 \%$ confidence interval for the RMSEA statistic was .041; this suggests the three-factor solution has an excellent fit to the data.

\section{Relation of RRB factors to other subject characteristics}

The effects of age, IQ, and social and communication deficits (as measured by the Social and Communication Domain scores on the ADIR) on RRB factor scores were examined using Pearson correlations. ANOVAs were run to examine the effects of categorical variables (sex and Loss of Skills before Age 5 [as an indication of regression as measured by the ADI-R item 95; scored ' 0 ' = no loss, ' 1 ' possible loss; '2' definite loss]) on RRB factor scores. Results are in Tables 3 and 4. Overall, higher scores on the RMB factor were associated with younger age, lower verbal IQ, greater social deficits, greater communication impairments (in verbal subjects), and loss of skills. Post-hoc Tukey analysis showed that for loss of skills, those with either a possible or definite loss had higher levels of motor stereotypies than those who had no loss whatsoever. Higher scores on the IS factor were associated only with greater communication impairments in verbal subjects and greater social deficits. These correlations, while statistically significant, are relatively small in magnitude. Interestingly, the CI factor did not show any significant relations to any of these subject variables.

\section{Familiality of RRB factors: sib-pair data}

In order to examine potential familiality of RRB, intraclass correlations (ICCs) were computed for each of the three factors (RMB, IS, and CI) for 126 sib-pairs. Both siblings had a diagnosis of autistic disorder. No evidence of significant familial aggregation was shown for the RMB factor (ICC $=.17, p=.06$ ). However, significant ICCs were found for both IS $(.33, p<.001)$ and $\mathrm{CI}(.31, p<.001)$, suggesting potential genetic mechanisms underlying their expression.

\section{Using RRB factors to sort cases}

To examine the data at the case level (to start to address the clinical heterogeneity of autism), participants were sub-grouped into one of four categories based on their RRB factor scores: (1) elevated levels of all three RRB factors $(N=28,8.9 \%)$, (2) elevated levels of two RRB factors $(N=56,17.7 \%)$, (3) elevated level on one RRB factor $(N=96,30.4 \%)$, or (4)

\footnotetext{
${ }^{1}$ This item is not scored in children under the age of 3 ; for those cases, their data for that item was treated as missing and CI factor scores were not computed.
} 
no elevated levels of any of the RRB factors $(N=40,12.7 \%)$. A median-split was used to determine whether or not a subject would be classified as having an 'elevated' level on any given factor. If a subject was missing a factor score due to missing data, they were not assigned to a group for these analyses ( $N=96,30.4 \%$ of the sample). The four RRB groups then were compared to one another to see if they differed systematically on gender or the presence of regression using chi squares analysis, which showed no differences (Gender, $X^{2}$ $(3,211)=1.80, p=.61, p=.28$; Regression, $\left.X^{2}(6,211)=7.46, p=.28\right)$. Next, ANOVAs were run to see the effect of RRB group on social and communication deficits (as measured by the Social and Communication Domain scores on the ADI-R). ${ }^{2}$ Results indicated a significant effect of RRB group on both domains (Social, $F(3,217)=3.05, p<.05$; Communication in Verbal Subjects, $F(3,157)=6.17, p<.01)$. Post-hoc analyses revealed that the presence of at least one elevated RRB factor was associated with more severe social impairment, and the presence of at least two elevated RRB factors was associated with more severe communication impairment in verbal subjects (see Figure 1).

\section{Discussion}

Although classically considered a unitary domain of behavior, the current study adds to a growing body of work suggesting meaningful subtypes of restricted repetitive behavior exist in autism. Most of these studies have suggested two potential subtypes of RRB ('lower order' RSMB and 'higher order' IS). The current study closely replicates these two factors (our IS factor is identical to those found by Cuccaro et al., (2003), Shao et al., (2003), and Szatmari et al. [2006] and our RMB factor is quite similar to these previous studies with the exception of the removal of the 'Unusual Sensory Interests' item). However, we also found evidence for an additional factor, Circumscribed Interests. To examine whether or not our novel finding was due to our exclusion of the sensory item, we ran the PCA and EFA again with 'Unusual Sensory Interests' included, which still revealed a three-factor solution with a distinct CI factor. We also repeated our factor analyses on the 'ever' ratings, which also replicated the three-factor solution reported here. Therefore, our data strongly suggest that circumscribed interests are a distinct subgroup of repetitive behavior in autism, which may have potentially important implications in both research and clinical settings.

Circumscribed interests, as identified in this study, include behaviors such as intense, focused hobbies, strong preoccupations with odd topics (such as sewer systems or garage doors), and unusually strong attachment to certain objects. This is similar to previous descriptions of circumscribed interests (e.g., Boyd, Conroy, Mancil, Nakao, \& Alter, 2007; South, Ozonoff, \& McMahon, 2005) with the exception of our inclusion of object attachment. Our findings are in line with previous research examining the structure of RRB using the RBS-R (Repetitive Behavior Scale Revised; Bodfish et al., 2000) where an empirically-derived restricted/circumscribed interest factor was revealed, which also contained both verbally and non-verbally mediated focused interests, including object attachment (Lam \& Aman, 2007). Perhaps the essential element of CI is the limited range of focus, interest, or activity, and is not confounded by the verbal capabilities of the individual. This may allow us to identify CI in a broad range of cases; this is consistent with previous research that has shown that circumscribed interests can be found in higher- and lowerfunctioning individuals with autism (Bartak \& Rutter, 1976; Freeman et al., 1981).

Unlike other restricted repetitive behaviors which are found among a variety of genetic, developmental and psychiatric disorders, we are unaware of any disorders outside the autism spectrum that include circumscribed interests as a manifestation of repetitive behaviors.

\footnotetext{
${ }^{2}$ The effect of RRB group on IQ data could not be examined due to the fact that IQ data was only available on a subset of cases in each group.
} 
However, relatively little research has examined these behaviors in detail. In a recent study of 40 children and adolescents with high-functioning autism and Asperger's syndrome, South and colleagues (2005) examined 4 types of repetitive behaviors as defined in the DSM-IV: (1) Repetitive Object Use, (2) Motor Movements, (3) Rigid Routines, and (4) Circumscribed Interests. They found an increase in circumscribed interests over time while the other types of RRB peaked in severity earlier in life. Although this increase in CI was not observed in the current sample, this may be due to a couple of factors. First, our sample had an average age of 9.02 years at the time of the ADI-R administration; considerably younger than the sample reported by South et al., which had a mean age of 14 years (South et al., 2005). It is possible that an increase in CI may occur in adolescence and early adulthood. In addition, the lack of an age effect in the current sample may also be due to a limitation of the ADI-R to measure the full breadth and severity of circumscribed interests, so further research is needed. If CI do show a unique developmental course relative to other types or RRB, this would provide further evidence that CI may be a distinct subtype of behavior.

It has been postulated that CI may interfere with the development of peer relationships due to the fact that the individual only cares to converse with others about his/her interest (Atwood, 1998). In severe cases, individuals with CI may not be able to inhibit the preoccupations in order to participate in school or home life, which could contribute to academic and adaptive delays. Parents of children with ASD have also reported that CI symptoms, especially incessant talking about a certain topic, were among the most difficult aspects of autism they had to deal with on a daily basis (South et al., 2005). More research is needed on the specificity of CI to autism, and given that they are often an area of clinical concern, the ability to distinctly measure them may be of use to researchers and clinicians (Boyd et al., 2007).

When examining the relations between the three RRB factors and other subject characteristics (such as IQ, sex, age, and loss of skills/regression), several things became clear. First, each of the three RRB factors was differentially related to these subject variables, which serves as an additional validity check that they are indeed measuring distinct aspects of the behavioral phenotype. The RMB factor was most closely associated with other subject characteristics; significant relations were found with younger age, lower verbal IQ, greater social deficits, greater communication impairments (in verbal subjects), and loss of language. These findings are similar to those of Hus and colleagues (2007), who found that these behaviors were correlated with verbal and nonverbal IQ and all symptom areas on the ADI-R.Cuccaro et al. (2003) and Bishop et al. (2006) also found significant relations between RSMA scores and adaptive behavior and IQ.

In the present study, our IS factor showed significant correlations with both the social and communication domains on the ADI-R, indicating that it may be tied to overall severity of autism in our sample. This is in line with the findings of Hus et al. (2007), who found similar statistically significant (albeit weak) correlations between the IS factor and ADI-R social and communication domains. Szatmari and colleagues (2006) found the IS factor to be associated with the ADI-R communication domain only. Lastly, the CI factor in the present study was shown to be quite independent; no significant relations were found between this factor score and sex, age, IQ, presence of language loss/regression, and autism symptom domains on the ADI-R.

Examination of the sib-pair data revealed significant familial associations for both the CI and IS factors, but not for the RMB factor. Overall, our results are consistent with previous research showing clear familial aggregation of the IS factor but not of the RSMB factor (Cuccaro et al., 2003; Szatmari et al., 2006). Our findings suggest that both the CI and IS 
factors may be of use in genetic investigation. However, the independence of the CI factor suggests that it may be of particular use in stratifying samples.

To begin to address the clinical heterogeneity of autism, we examined the presence of repetitive behavior at the case level to see if the presence of multiple types of RRB was associated with other features of autism. In order to maximize power for the analyses, we employed a median split to identify individuals with elevated levels of RRB. Future studies may want to examine more stringent criteria to classify more clinically-meaningful subgroups. However, these preliminary findings suggest that individuals that present with multiple types of RRBs tend to have more severe impairments in the social and communication domains. Current diagnostic guidelines (DSM-IV; APA, 1994) require the presence of only one restricted repetitive behavior for a diagnosis of autism. However, it is interesting to revisit Kanner's original report on autism where he described a series of children who, in addition to social and communication impairments, showed a variety of restricted repetitive behaviors (Kanner, 1943). Perhaps it is this co-occurrence of multiple forms of RRB (e.g., stereotyped body movements, the need for sameness, and intense interests) that distinguishes more severe, 'Kannerian' autism from milder variants that still meet current diagnostic criteria.

\section{Limitations/Future directions}

A major limitation of this study is the use of the ADI-R as the sole measure of restricted repetitive behavior. The ADI-R is a diagnostic parent interview, and although it has been shown to be a reliable and valid way of identifying children with autism (e.g., Lord et al., 1997, 1994), it was not intended as a measure of RRB in any detail. In fact, some work has suggested that the domain of restricted repetitive behavior is undersampled by the ADI-R (Lecavalier et al., 2006). Therefore, our findings must be viewed as preliminary, and future studies should examine this domain in more detail, using questionnaires designed to capture the full breadth of RRB in autism (e.g., the RBS-R). In addition, direct observational studies of repetitive behaviors are needed.

\section{Acknowledgments}

Supported By: NIH T32 HD40127, NIH R01 MH73402, R01MH55284. R01MH61696, U54MH66418, R29 MH51217.

\section{References}

American Psychiatric Association. Diagnostic and statistical manual of mental disorders. 4th edn, text rev.. Washington, DC: APA; 1994.

Arthur, G. The Arthur Adaptation of the Leiter International Performance Scale. Chicago: Stoelting; 1952.

Atwood, T. Asperger's Syndrome: A guide for parents and professionals. Philadelphia: Taylor \& Francis; 1998.

Baranek GT, David FJ, Poe MD, Stone WL, Watson LR. Sensory Experiences Questionnaire: Discriminating sensory features in young children with autism, developmental delays, and typical development. Journal of Child Psychology and Psychiatry. 2006; 47:591-601. [PubMed: 16712636]

Barrett S, Beck JC, Bernier R, Bisson E, Braun TA, Casavant TL, Childress D, Folstein SE, Garcia M, Gardiner MB, Gilman S, Haines JL, Hopkins K, Landa R, Meyer NH, Mullane JA, Nishimura DY, Palmer P, Piven J, Purdy J, Santangelo SL, Searby C, Sheffield V, Singleton J, Slager S, Struchew T, Svenson S, Vieland V, Wong K, Winklosky B. An autosomal genomic screen for autism. Collaborative linkage study of autism. American Journal of Medical Genetics. 1999; 88:609-615. Erratum in: American Journal of Medical Genetics, 2001, December 8, 105, 805. [PubMed: 10581478] 
Bartak L, Rutter M. Differences between mentally retarded and normally intelligent autistic children. Journal of Autism and Childhood Schizophrenia. 1976; 6:109-120. [PubMed: 989485]

Bishop SL, Richler J, Lord C. Association between restricted and repetitive behaviors and non-verbal IQ in children with autism spectrum disorders. Child Neuropsychology. 2006; 12:247-267. [PubMed: 16911971]

Bodfish JW, Symons FJ, Parker DE, Lewis MH. Varieties of repetitive behavior in autism: Comparisons to mental retardation. Journal of Autism and Developmental Disorders. 2000; 30:237243. [PubMed: 11055459]

Boyd BA, Conroy MA, Mancil GR, Nakao T, Alter PJ. Effects of circumscribed interests on the social behaviors of children with autism spectrum disorders. Journal of Autism and Developmental Disorders. 2007; 37:1550-1561. [PubMed: 17146704]

Bradford Y, Haines J, Hutcheson H, Gardiner M, Braun T, Sheffield V, et al. Incorporating language phenotypes strengthens evidence of linkage to autism. American Journal of Medical Genetics. 2001; 105:539-547. [PubMed: 11496372]

Browne MW. An overview of analytic rotation in exploratory factor analysis. Multivariate Behavioral Research. 2001; 36:111-150.

Browne MW, Cudeck R. Alternative ways of assessing model fit. Sociological Methods, and Research. 1992; 21:230-258.

Buxbaum JD, Silverman JM, Smith CJ, Kilifarski M, Reichert J, Hollander E, et al. Evidence for a susceptibility gene for autism on chromosome 2 and for genetic heterogeneity. American Journal of Human Genetics. 2001; 68:1514-1520. [PubMed: 11353400]

Cattell RB. The scree test for the number of factors. Multivariate Behavioral Research. 1966; 1:245276.

Cuccaro ML, Shao Y, Grubber J, Slifer M, Wolpert CM, Donnelly SL, et al. Factor analysis of restricted and repetitive behaviors in autism using the Autism Diagnostic Interview-R. Child Psychiatry and Human Development. 2003; 34:3-17. [PubMed: 14518620]

Fabrigar LR, Wegener DT, MacCallum RC, Strahan EJ. Evaluating the use of exploratory factor analysis in psychological research. Psychological Methods. 1999; 4:272-299.

Folstein SE, Piven J. Etiology of autism: Genetic influences. Pediatrics. 1991; 87(5 Pt 2):767-773. [PubMed: 1708492]

Freeman BJ, Ritvo ER, Schroth PC, Tonick I, Guthrie D, Wake L. Behavioral characteristics of highand low-IQ autistic children. American Journal of Psychiatry. 1981; 138:25-29. [PubMed: 7446777]

Hazlett HC, Poe M, Gerig G, Smith RG, Provenzale J, Ross A, Gilmore J, Piven J. Magnetic resonance imaging and head circumference study of brain size in autism: Birth through age 2 years. Archives of General Psychiatry. 2005; 62:1366-1376. [PubMed: 16330725]

Hazlett HC, Poe MD, Gerig G, Smith RG, Piven J. Cortical gray and white brain tissue volume in adolescents and adults with autism. Biological Psychiatry. 2006; 59:1-6. [PubMed: 16139816]

Hus V, Pickles A, Cook EH Jr, Risi S, Lord C. Using the Autism Diagnostic Interview-Revised to increase phenotypic homogeneity in genetic studies of autism. Biological Psychiatry. 2007; 61:438-448. [PubMed: 17276746]

Kanner L. Autistic disturbances of affective contact. Nervous Child. 1943; 2:217-250.

Lam KSL, Aman MG. The Repetitive Behavior Scale-Revised: Independent validation in individuals with autism spectrum disorders. Journal of Autism and Developmental Disorders. 2007; 37:855866. [PubMed: 17048092]

Lecavalier L, Aman MG, Scahill L, McDougle CJ, McCracken JT, Vitiello B, et al. Validity of the Autism Diagnostic Interview-Revised. American Journal on Mental Retardation. 2006; 111:199215. [PubMed: 16597187]

Lord C, Pickles A, McLennan J, Rutter M, Bregman J, Folstein S, et al. Diagnosing autism: Analyses of data from the Autism Diagnostic Interview. Journal of Autism and Developmental Disorders. 1997; 27:501-517. [PubMed: 9403369]

Lord C, Rutter M, Le Couteur A. Autism Diagnostic Interview-Revised: A revised version of a diagnostic interview for caregivers of individuals with possible pervasive developmental disorders. Journal of Autism and Developmental Disorders. 1994; 24:659-685. [PubMed: 7814313] 
Lord, C.; Rutter, M.; DiLavore, PC.; Risi, S. Autism Diagnostic Observation Schedule. Los Angeles, CA: Western Psychological Services; 2002.

Lotspeich LJ, Ciaranello RD. The neurobiology and genetics of infantile autism. International Reviews in Neurobiology. 1993; 35:87-129.

Mullen, EM. Mullen Scales of Early Learning: AGS edition. Circle Pines, MN: American Guidance Service; 1995.

Piven J, Palmer P, Landa R, Santangelo S, Jacobi D, Childress D. Personality and language characteristics in parents from multiple-incidence autism families. American Journal of Medical Genetics. 1997; 74:398-411. [PubMed: 9259376]

Rutter M. Incidence of autism spectrum disorders: Changes over time and their meaning. Acta Paediatrica. 2005; 94:2-15. [PubMed: 15858952]

Rutter, M.; Le Couteur, A.; Lord, C. ADI-R: The Autism Diagnostic Interview-Revised. Los Angeles, CA: Western Psychological Services; 2003.

Shao Y, Cuccaro ML, Hauser ER, Raiford KL, Menold MM, Wolpert CM, et al. Fine mapping of autistic disorder to chromosome 15q11-q13 by use of phenotypic subtypes. American Journal of Human Genetics. 2003; 72:539-548. [PubMed: 12567325]

Shao Y, Raiford KL, Wolpert CM, Cope HA, Ravan SA, Ashley-Koch AA, et al. Phenotypic homogeneity provides increased support for linkage on chromosome 2 in autistic disorder. American Journal of Human Genetics. 2002; 70:1058-1061. [PubMed: 11875756]

South M, Ozonoff S, McMahon W. Repetitive behavior profiles in Asperger syndrome and highfunctioning autism. Journal of Autism and Developmental Disorders. 2005; 35:145-158. [PubMed: 15909401]

Szatmari P, Georgiades S, Bryson S, Zwaigenbaum L, Roberts W, Mahoney W, et al. Investigating the structure of the restricted, repetitive behaviours and interests domain of autism. Journal of Child Psychology and Psychiatry. 2006; 47:582-590. [PubMed: 16712635]

Veenstra-Vanderweele J, Christian SL, Cook EH Jr. Autism as a paradigmatic complex genetic disorder. Annual Review of Genomics and Human Genetics. 2004; 5:379-405.

Wassink TH, Piven J, Vieland VJ, Pietila J, Goedken RJ, Folstein SE, et al. Examination of AVPR1a as an autism susceptibility gene. Molecular Psychiatry. 2004; 9:968-972. [PubMed: 15098001]

Wechsler, D. Wechsler Adult Intelligence Scale-Revised. San Antonio, TX: The Psychological Corporation; 1981.

Wechsler, D. Wechsler Intelligence Scale for Children. 3rd edn. San Antonio, TX: The Psychological Corporation; 1991.

World Health Organization. International statistical classification of diseases and related health problems. 10th edn. Vol. vol. 10. Geneva: World Health Organization; 1992. 




Figure 1.

Levels of social and verbal communication impairment by RRB grouping 
Table 3

Correlations between subject characteristics and RRB factor scores

\begin{tabular}{lrrr}
\hline & \multicolumn{3}{c}{$\begin{array}{c}\text { Pearson correlations } \\
\text { (for continuous variables) }\end{array}$} \\
\cline { 2 - 5 } & $\mathbf{R M B}$ & IS & CI \\
\hline Age & $-.41^{* *}$ & .08 & .05 \\
Verbal IQ & $-.46^{* *}$ & .09 & .04 \\
Performance IQ & .09 & -.18 & -.14 \\
Social deficits & $.21^{* *}$ & $.15^{*}$ & .07 \\
Communication deficits: Verbal subjects & $.22^{* *}$ & $.26^{* *}$ & .15 \\
Communication deficits: Nonverbal subjects & .18 & -.06 & -.10 \\
\hline$*$ & & & \\
$p<0.05 ;$ & & & \\
$* *$ \\
$p<0.01$.
\end{tabular}


